\title{
Universiteit
}

Leiden

The Netherlands

\section{Oxygen-enhanced atomic chain formation}

Thijssen, W.H.A.; Marjenburgh, D.; Bremmer, R.H.; Ruitenbeek, J.M. van

\section{Citation}

Thijssen, W. H. A., Marjenburgh, D., Bremmer, R. H., \& Ruitenbeek, J. M. van. (2006). Oxygenenhanced atomic chain formation. Physical Review Letters, 96, 026806.

doi:10.1103/PhysRevLett.96.026806

Version: $\quad$ Not Applicable (or Unknown)

License: $\quad$ Leiden University Non-exclusive license

Downloaded from: https://hdl.handle.net/1887/62238

Note: To cite this publication please use the final published version (if applicable). 


\title{
Oxygen-Enhanced Atomic Chain Formation
}

\author{
W. H. A. Thijssen, ${ }^{1}$ D. Marjenburgh, ${ }^{1}$ R. H. Bremmer, ${ }^{1}$ and J. M. van Ruitenbeek ${ }^{1}$ \\ ${ }^{1}$ Kamerlingh Onnes Laboratorium, Universiteit Leiden, Niels Bohrweg 2, 2333 CA Leiden, Netherlands
} (Received 15 September 2005; published 19 January 2006)

\begin{abstract}
We report experimental evidence for atomic chain formation during stretching of atomic-sized contacts for gold and silver, that is strongly enhanced due to oxygen incorporation. While gold has been known for its tendency to form atomic chains, for silver this is only observed in the presence of oxygen. With oxygen the silver chains are as long as those for gold, but the conductance drops with chain length to about 0.1 conductance quantum. A relation is suggested with previous work on surface reconstructions for silver (110) surfaces after chemisorption of oxygen.
\end{abstract}

DOI: 10.1103/PhysRevLett.96.026806

PACS numbers: 73.63.Rt, 68.35.-p, 73.40.Jn, 81.07.Lk

In recent years it has become possible to measure the electrical and mechanical properties of the ultimate one dimensional metallic conductor: a chain of single atoms. For gold, freely suspended single-atom chains up to seven or eight atoms long have been created and investigated [1-3] and it has been possible to identify the vibration modes by means of point contact spectroscopy [4]. It has been argued that the underlying physical mechanism for the formation of monatomic chains for $\mathrm{Au}, \mathrm{Pt}$, and $\mathrm{Ir}$ is the same as for the surface reconstructions on clean [110] surfaces of these $5 d$ metals [5]. The related $4 d$ metals, such as Ag in the case of Au, were found to form only short chains in low temperature break junction experiments, just two, or rarely three, atoms long [5,6]. Indeed, Ag does not show the same tendency to surface reconstructions. However, when the [110] surface reacts with oxygen it does reconstruct into an added row reconstruction with -Ag-O-Ag- rows lying in the [001] direction on the surface as seen by STM studies [7,8].

Previous transmission electron microscopy (TEM) measurements on suspended Au chains, created at room temperature and under ultrahigh vacuum (UHV) conditions have shown anomalously large interatomic distances of up to $4 \AA$ [2], compared to $2.9 \AA$ in bulk Au. On the other hand, Au atomic wires created under cryogenic vacuum at $4.2 \mathrm{~K}$ reveal an interatomic distance of $2.5 \pm 0.2 \AA$ [9], which is consistent with calculations on the formation of $\mathrm{Au}$ wires [10-12]. By a similar room temperature TEM method atomic $\mathrm{Ag}$ chains with large interatomic distances have been observed as well [13], which is at variance with low temperature break junction results [6]. It has therefore been suggested that the observation of these large interatomic distances may be due to the incorporation of light foreign atoms, which cannot be imaged by TEM [14-16]. Recent density functional theory calculations have predicted that oxygen can be stably incorporated into a $\mathrm{Au}$ chain $[17,18]$. The Au-O bond was found to be stronger than a bulk Au-Au bond, and even induces a strengthening of the neighboring Au-Au bond in the chain, which would imply that longer chains may also be formed. The con- ductance of these Au-O chains should stay close to 1 conductance quantum $G_{0}=2 e^{2} / h$ [18], as is the case for a pure Au chain. In this Letter we discuss experiments on admitting oxygen to atomic chains of $\mathrm{Au}$ and $\mathrm{Ag}$ atoms. We present evidence that oxygen chemisorbs to the atomic chains, and stabilizes the atomic chains by being incorporated. For Ag the presence of oxygen allows the formation of chains as long as those for Au. Our observations open the way to investigate mixed atomic wires with inclusions of foreign atoms or molecules.

The experiments were done using the mechanically controllable break junction technique (see Ref. [19] for a detailed description). The sample wire is first broken under cryogenic vacuum at $4.2 \mathrm{~K}$, in order to create clean fracture surfaces. The temperature of the sample can be varied using a local heater and thermometer, while the rest of the chamber remains at $4.2 \mathrm{~K} . \mathrm{O}_{2}$ can be admitted via a capillary, equipped with a heater wire running all along its interior that prevents premature condensation of the gas. The amount introduced is of order $10 \mu \mathrm{mol}$, but the dose arriving at the atomic contact cannot be determined very precisely.

When a $\mathrm{Au}$ or $\mathrm{Ag}$ wire is broken gently, a thin neck is being formed between the two contact leads. By further stretching of the contact the neck is thinned and finally a contact of only a single atom is formed. During the final stages of breaking one observes a steplike decrease of the conductance and a last plateau that has a conductance near $1 G_{0}$. Figure 1 shows typical conductance traces for clean $\mathrm{Au}$ and $\mathrm{Ag}$, and for $\mathrm{Au}$ and $\mathrm{Ag}$ after admitting $\mathrm{O}_{2}$. The last plateau of a breaking trace for clean $\mathrm{Au}$ can often be stretched to quite long lengths, which signals the formation of monatomic chains [1]. By collecting a large set of these traces a conductance histogram can be constructed. Both for $\mathrm{Au}$ and $\mathrm{Ag}$ at $4.2 \mathrm{~K}$ the conductance histogram is dominated by a single sharp peak at $1 G_{0}$, indicating that a contact of a single atom in cross section is occurring frequently (Fig. 2). After admitting $\mathrm{O}_{2}$ to $\mathrm{Au}$ and $\mathrm{Ag}$ atomic contacts at $4.2 \mathrm{~K}$ the conductance for $\mathrm{Au}$ does not significantly change, but for $\mathrm{Ag}$ a dominant peak around 


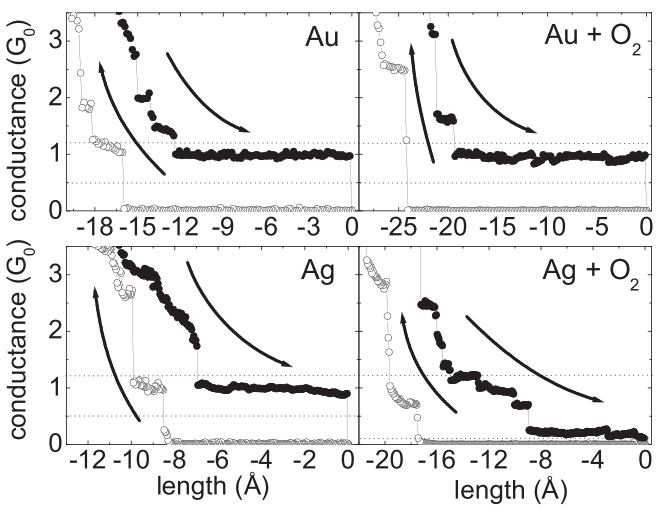

FIG. 1. Individual breaking and return traces for clean $\mathrm{Au}$, for $\mathrm{Au}$ after admitting $\mathrm{O}_{2}$, for $\mathrm{Ag}$, and for $\mathrm{Ag}$ after admitting $\mathrm{O}_{2}$. A bias voltage of $50 \mathrm{mV}$ was used in all cases.

$0.1 G_{0}$ appears together with a large background, indicating that stable configurations with conductances below $1 G_{0}$ are regularly formed [Fig. 2(c)]. As the histograms have been normalized to the area under the curve one can see that the weight of the peak at $1 G_{0}$ has been transferred to the structure at lower conductances. When clean Au is heated to $40 \mathrm{~K}$ the average chain length decreases, which can be seen from the lower relative weight of the peak at $1 G_{0}$ in Fig. 2(b). However, when $\mathrm{O}_{2}$ is admitted the peak around $1 G_{0}$ becomes more prominent and, in addition, a peak at $0.1 G_{0}$ appears. On the other hand, for $\mathrm{Ag}$ with $\mathrm{O}_{2}$ at $40 \mathrm{~K}$ only the background is further increased as compared to $4.2 \mathrm{~K}$.

By repeating the process of breaking and making a contact several thousands of times and automatically recording the lengths of the last plateaus near $1 G_{0}$ one can

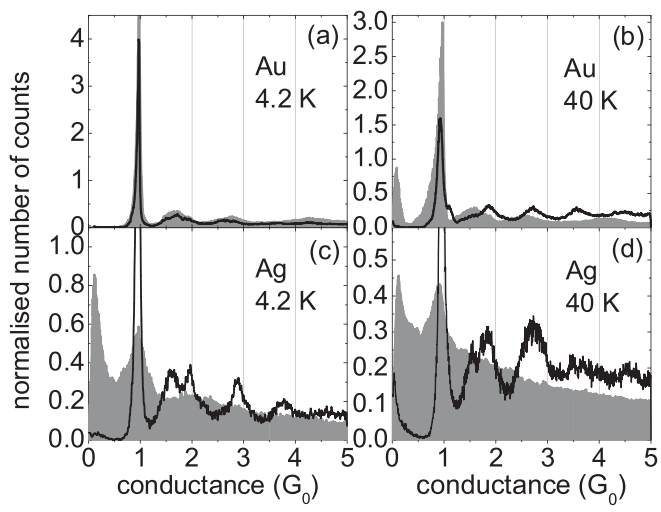

FIG. 2. Conductance histograms for (a) Au (black curve) and $\mathrm{Au}$ after admitting $\mathrm{O}_{2}$ (filled graph) at $4.2 \mathrm{~K}$; (b) $\mathrm{Au}$ (black curve) and $\mathrm{Au}$ after admitting $\mathrm{O}_{2}$ (filled graph) at $40 \mathrm{~K}$; (c) $\mathrm{Ag}$ (black curve) and $\mathrm{Ag}$ after admitting $\mathrm{O}_{2}$ (filled graph); (d) $\mathrm{Ag}$ (black curve) and $\mathrm{Ag}$ after admitting $\mathrm{O}_{2}$ at $40 \mathrm{~K}$. Because of scaling the $1 G_{0}$ peak maxima for clean $\mathrm{Ag}$ in (c) and (d) are not seen; maximum values are 2.2 and 1.8, respectively. A bias voltage of $50 \mathrm{mV}$ was used and each graph is built from approximately 2000 breaking traces. construct a length histogram. The length starts to be recorded when the conductance drops below $1.2 G_{0}$ and stops for $G<0.5 G_{0}$. A typical length histogram for Au recorded at $4.2 \mathrm{~K}$ reveals several equidistant peaks that decrease in height exponentially after the first two peaks, as can be seen from the black curve in Fig. 3(a). The distance between the peaks gives the interatomic distance of the atoms in the chain $[1,9]$. Numerical simulations show that the conductance of a Au atomic contact drops just below $1 G_{0}$ when both contact apexes end in a single atom [20-22]. Starting from that configuration the length of the chain is being recorded in our experiments. The position of the first peak in the histogram can thus be interpreted as being the difference in length between a one atom contact and a chain of two atoms, both at the point of breaking. The position of the second peak is the difference in length between a one atom contact and a chain consisting of three Au atoms, etc., The histogram shows that chains of two and three atoms long have a high probability to be formed and that the probability for the formation of longer chains decreases very rapidly.

After $\mathrm{O}_{2}$ is admitted to the contact while it is kept at $4.2 \mathrm{~K}$, a small but clearly observable change in the distance between some peaks in the length histogram is observed [Fig. 3(a)]. Some of the peaks of the filled graph (Au with $\mathrm{O}_{2}$ ) are shifted somewhat to the left compared to the peaks of the black curve (clean $\mathrm{Au}$ ), notably the second peak and the peaks that are made visible in the inset of Fig. 3(a). A closer look at the distances in Fig. 3(a) observed for $\mathrm{Au}$ with $\mathrm{O}_{2}$, suggests two sets of distances, namely $2.5 \pm$ $0.2 \AA$, seen, e.g., between the second and third peak, and

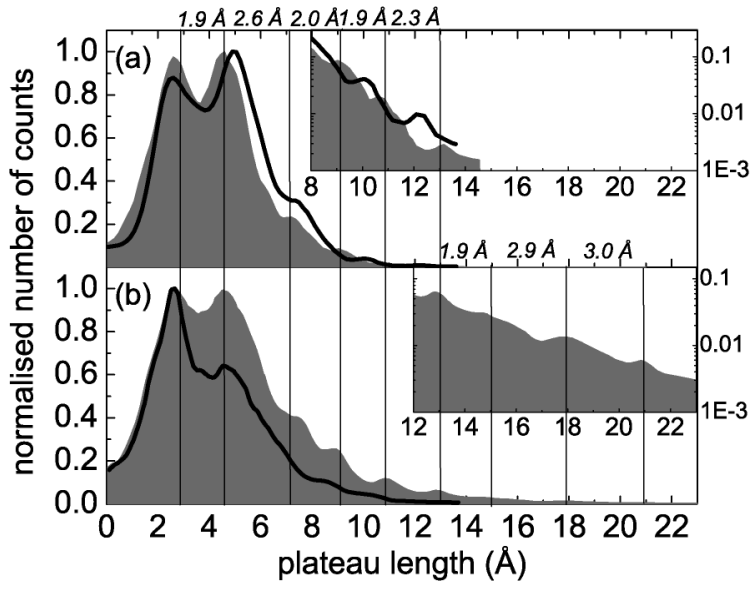

FIG. 3. Panels (a) and (b) show length histograms for $\mathrm{Au}$ (black curve) and for $\mathrm{Au}$ after admitting $\mathrm{O}_{2}$ at $4.2 \mathrm{~K}$ and $40 \mathrm{~K}$, respectively. The insets show semilog plots of the tails of the distributions in order to bring out the small peaks. Vertical lines are shown at the peak positions in the length histograms for $\mathrm{Au}$ with $\mathrm{O}_{2}$, together with their distances. In all cases a bias voltage of $50 \mathrm{mV}$ was used and the start and stop conductance values between which the lengths of the plateaus were measured were $1.1 G_{0}$ and $0.5 G_{0}$, respectively. 
$1.9 \pm 0.2 \AA$, seen, e.g., between the first and second peak. When the temperature of a clean Au contact is raised to $40 \mathrm{~K}$ a faster decrease of the intensity of the peaks is seen, as the result of thermal decay of the chain configuration; see Fig. 3(b) (black curve). When at $40 \mathrm{~K} \mathrm{O}_{2}$ gas is admitted a remarkable observation is made. Again the distances between some peaks change but the average length of the chains is significantly longer, even longer than at $4.2 \mathrm{~K}$. Chains up to ten atoms long are observed, as seen in Fig. 3(b).

Even more spectacular results are obtained when performing these same experiment for Ag. A length histogram for $\mathrm{Ag}$ recorded at $4.2 \mathrm{~K}$ is shown as the black curve in Fig. 4(a). $\mathrm{O}_{2}$ was admitted when the Ag atomic contact was kept at $4.2 \mathrm{~K}$. Since a peak around $0.1 G_{0}$ appears (see Fig. 2) the start and stop conductance values for measuring the chain length were put at $1.2 G_{0}$ and $0.05 G_{0}$ respectively. It is very striking that the average chain length is dramatically increased compared to a clean $\mathrm{Ag}$ contact. A sequence of peaks is observed indicating the repeated occurrence of certain stable chain configurations. When a chain of single atoms breaks its conductance usually drops to a very low conductance, deep into the tunneling regime as a result of the collapse of the atoms back to the leads. In the lower right panel of Fig. 1 a typical breaking trace and

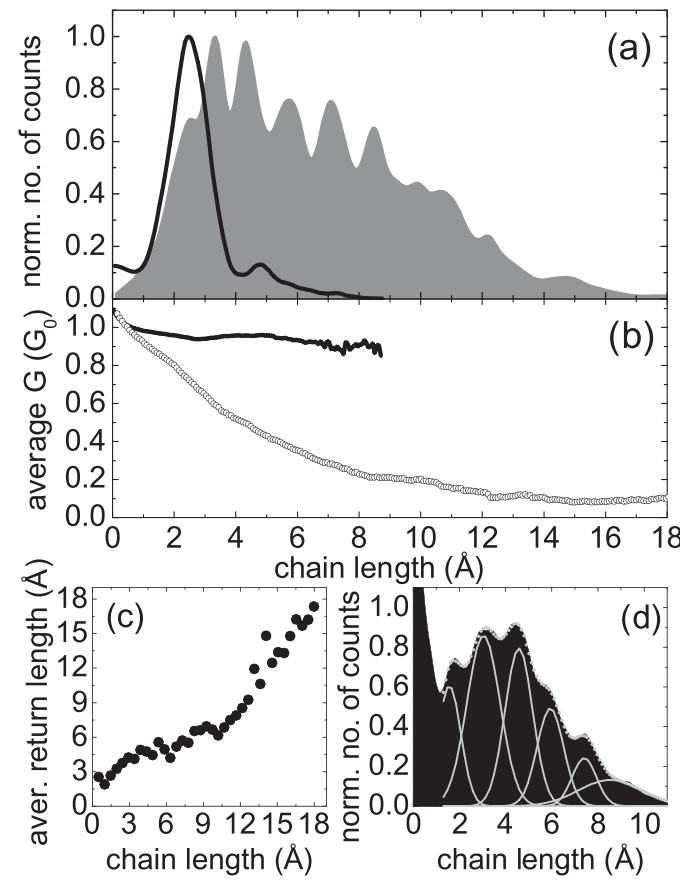

FIG. 4. (a) Length histogram for clean Ag (black curve) and for $\mathrm{Ag}$ after admitting $\mathrm{O}_{2}$ (filled graph) at $4.2 \mathrm{~K}$. (b) Average conductance as a function of chain length for clean Ag (black curve) and for $\mathrm{Ag}$ with $\mathrm{O}_{2}$ (open circles). (c) Average return lengths as a function of Ag-O chain lengths. (d) Length histogram of $\mathrm{Ag}$ after admitting $\mathrm{O}_{2}$ with start and stop values of $0.5 G_{0}$ and $0.05 G_{0}$, respectively. its return trace for $\mathrm{Ag}$ with $\mathrm{O}_{2}$ are shown. The return trace shows the conductance of the contact when it is being made again after it has been broken. The observation of additional plateaus with conductance values as low as $0.1 G_{0}$ indicates that the chain is still mechanically intact. We have verified that the conductance plateaus are indeed due to chain formation by measuring the average return length as a function of the length of the chains. The return length is the distance over which the electrodes need to be moved in order to return to contact. We confirm that these two lengths are, on average, nearly the same; see Fig. 4(c). Figure 4(b) shows the average conductance of the clean Ag chains and for Ag-O chains as function of chain length. The curves are obtained by adding all measured conductance traces from the start value $\left(1.2 G_{0}\right)$ onward, and dividing at each length by the number of traces included at that point. For clean $\mathrm{Ag}$ the conductance remains close to $1 G_{0}$, with small parity oscillations with the number of atoms in the chain, as was previously observed for $\mathrm{Au}$ [3]. In contrast, the average conductance for Ag-O chains decreases rapidly as the chains become longer, stabilizing at approximately $0.1 G_{0}$. Further evidence for chains incrementing by discrete atomic units can be found from Fig. 4(d), which shows a length histogram for $\mathrm{Ag}$ with $\mathrm{O}_{2}$ for which the start and stop values were set to $0.5 G_{0}$ and $0.05 G_{0}$, respectively. At least five peaks can clearly been seen and the histogram is perfectly fitted with a multiple Gaussian distribution that again gives an interpeak distance of $1.5 \pm 0.2 \AA$. Similar results were obtained when these experiments on $\mathrm{Ag}-\mathrm{O}$ chain were repeated at $40 \mathrm{~K}$.

In order to interpret these unexpected results we consider the possibility of atomic oxygen being incorporated into $\mathrm{Au}$ and $\mathrm{Ag}$ chains. It is known that low-coordinated $\mathrm{Au}$ atoms are chemically very reactive [23,24]. As Au atoms have a coordination number of only two an atomic chain is expected to be even more reactive then a nanoparticle, opening the possibility for molecular oxygen to dissociate, even at low temperatures. The oxygen atoms are then expected to be incorporated in the Au chain, as predicted $[17,18]$. We have seen that the peak distances in the length histogram decrease upon admitting oxygen. The distance of $1.9 \AA$ agrees with the Au-O bond in the calculations $[17,18]$. At $40 \mathrm{~K}$ considerably longer chains are formed after admitting $\mathrm{O}_{2}$, which agrees with the Au-O bond being stronger than the $\mathrm{Au}-\mathrm{Au}$ bond $[17,18]$. We expect that at $4.2 \mathrm{~K}$ an $\mathrm{O}_{2}$ molecule can only be dissociated when it lands directly on the atomic chain. The fact that peaks are observed in the length histogram suggests that specific chain compositions are preferentially formed. The observed chain compositions can be explained by assuming only one $\mathrm{O}_{2}$ molecule that has dissociated on the $\mathrm{Au}$ chain, suggesting that at $4.2 \mathrm{~K}$ only two oxygen atoms are typically incorporated in the chain. When the temperature is increased to $40 \mathrm{~K}$ the efficiency for dissociating oxygen will be enhanced, making more oxygen atoms available for 
building a long chain. This chain is reinforced relative to a clean $\mathrm{Au}$ chain.

In the case of $\mathrm{Ag}$ with $\mathrm{O}_{2}$ we observe a distance between peaks of $1.5 \pm 0.2 \AA$ and long chains even at $4.2 \mathrm{~K}$, suggesting that low-coordinated $\mathrm{Ag}$ atoms dissociate $\mathrm{O}_{2}$ molecules more readily, in agreement with the higher reactivity of Ag.

We conclude that oxygen atoms are incorporated in chains of $\mathrm{Au}$ or $\mathrm{Ag}$ atoms, thus reenforcing it, which is most apparent in the case of Ag. We suspect a fundamental connection between the mechanism for oxygen induced surface reconstructions on $\operatorname{Ag}(110)$ [7] and the strongly enhanced chain formation. The fact that oxygen chemisorption leads to similar metal-oxygen row-reconstructed surfaces for other metals [8] opens possibilities for further investigation of enhanced chain formation due to oxygen incorporation. Preliminary results for $\mathrm{Cu}$ confirm that oxygen also induces long $\mathrm{Cu}-\mathrm{O}$ chain formation. The mere observation that foreign atoms and molecules can chemically react locally at an atomic contact and even be incorporated into an atomic chain opens new possibilities for modifying and investigating different combinations of metal contacts and chains with foreign molecules and atoms. It may even be possible to exploit atomically thin wires to contact single molecules which would provide ideal quantum leads to the nano-object of study.

We thank P. Jelínek and F. Flores for valuable discussions and for communicating unpublished work. This work is part of the research program of the "Stichting FOM," partially financed through the SONS programme of the European Science Foundation, which is also funded by the European Commission, Sixth Framework Programme, and was also supported by the European Commission TMR Network program DIENOW.

[1] A. I. Yanson, G. Rubio Bollinger, H.E. van den Brom, N. Agraï, and J.M. van Ruitenbeek, Nature (London) 395, 783 (1998).

[2] H. Ohnishi, Y. Kondo, and K. Takayanagi, Nature (London) 395, 780 (1998).
[3] R. H. M. Smit, C. Untiedt, G. Rubio-Bollinger, R. C. Segers, and J. M. van Ruitenbeek, Phys. Rev. Lett. 91, 076805 (2003).

[4] N. Agraï, C. Untiedt, G. Rubio-Bollinger, and S. Vieira, Phys. Rev. Lett. 88, 216803 (2002).

[5] R.H. M. Smit, C. Untiedt, A. I. Yanson, and J. M. van Ruitenbeek, Phys. Rev. Lett. 87, 266102 (2001).

[6] R. H. M. Smit, Ph.D. thesis, Univeriteit Leiden 2003.

[7] M. Taniguchi, K. Tanaka, T. Hashizume, and T. Sakurai, Surf. Sci. Lett. 262, L123 (1992).

[8] F. Besenbacher and J. K. Nørskov, Prog. Surf. Sci. 44, 5 (1993).

[9] C. Untiedt et al., Phys. Rev. B 66, 085418 (2002).

[10] S. R. Bahn, N. Lopez, J. K. Nørskov, and K. W. Jacobsen, Phys. Rev. B 66, 081405 (2002).

[11] H. Häkkinen, R. N. Barnett, A. G. Scherbakov, and U. Landman, J. Phys. Chem. B 104, 9063 (2000).

[12] E. Z. da Silva, A. J. R. da Silva, and A. Fazzio, Phys. Rev. Lett. 87, 256102 (2001).

[13] V. Rodrigues, J. Bettini, A. R. Rocha, L. G. C. Rego, and D. Ugarte, Phys. Rev. B 65, 153402 (2002).

[14] S. B. Legoas, D. S. Galvão, V. Rodrigues, and D. Ugarte, Phys. Rev. Lett. 88, 076105 (2002).

[15] F. D. Novaes, A. J.R. da Silva, E.Z. da Silva, and A. Fazzio, Phys. Rev. Lett. 90, 036101 (2003).

[16] N. V. Skorodumova and S. I. Simak, Phys. Rev. B 67, 121404(R) (2003).

[17] F. D. Novaes, A. J. R. da Silva, E. Z. da Silva, and A. Fazzio, Phys. Rev. Lett. 96, 016104 (2006).

[18] S. R. Bahn, N. Lopez, J. K. Nørskov, and K. W. Jacobsen, Phys. Rev. B 66, 081405(R) (2002).

[19] N. Agraï, A. Levy Yeyati, and J.M. van Ruitenbeek, Phys. Rep. 377, 81 (2003).

[20] M. Dreher, F. Pauly, J. Heurich, J. C. Cuevas, E. Scheer, and P. Niebala, Phys. Rev. B 72, 075435 (2005).

[21] J. J. Palacios, A. J. Pérez-Jiménez, E. Louis, E. SanFabián, and J. A. Vergés, Phys. Rev. B 66, 035322 (2002).

[22] P. Jelínek, R. Pérez, J. Ortega, and F. Flores, Surf. Sci. 566, 13 (2004).

[23] M. Valden, X. Lai, and D. W. Goodman, Science 281, 1647 (1998).

[24] H. Häkinnen, R. N. Barnett, and U. Landman, J. Phys. Chem. B 103, 8814 (1999). 